\title{
X-ray Microanalysis of Light Elements
}

\author{
G.F. Bastin and H.J.M. Heijligers
}

Laboratory of Solid State and Materials Chemistry, University of Technology, P.O. Box 513, NL5600 MB Eindhoven, The Netherlands.

Quantitative x-ray microanalysis of the ultra-light elements boron, carbon, nitrogen, and oxygen by wavelength-dispersive methods requires a lot of dedication and rather complicated experimental procedures compared to the analysis of heavier $(Z>11)$ elements. The most difficult practical problems in the analysis are those related to the differences in the $\mathrm{x}$-ray emission profiles from one ultra-light element compound to another. These peak shape variations are the result of the fact that in exciting ultra-light element $\mathrm{x}$-rays electronic transitions of the bonding electrons are involved. As a consequence large peak shifts and peak shape alterations in WD analysis of ultra-light elements may occur [1]. Peak shifts can easily be accounted for by simply retuning the spectrometer when moving from standard to specimen. Peak shape alterations, however, are much more difficult to deal with because they force the operator to perform the intensity measurements in an integral fashion. The alterations in peak shape are most pronounced for the lightest element studied so far (boron) and they gradually decrease with increasing atomic number. They are also strongly dependent on the type of analyzer crystal used with its typical spectral resolution: synthetic multilayer crystals with their poorer spectral resolution exhibit less pronounced shape alteration effects than their conventional counterpart lead-stearate [2]. The analysis of boron presents additional problems because the shape of the $\mathrm{B}-\mathrm{K}_{\alpha}$ peak is found to be dependent also on the crystallographic orientation of the specimen with respect to the electron beam and the spectrometer. These peculiar effects must be attributed to the presence of polarized components in the $\mathrm{B}-\mathrm{K}_{\alpha}$ emission peak [3], which can partially be filtered out by the analyzer crystal. It is evident that all these effects together can make the analysis of an element such as boron decidedly tricky and the operator should at least be fully aware of all the problems associated with the analysis of the particular light element at hand.

Thanks to realistic $\varphi(\rho z)$ approaches modern matrix correction procedures are no longer a limiting factor in the analysis. An often underestimated, but major, physical problem in the quantification of ultra-light elements, however, is the uncertainty in the mass absorption coefficients. It can easily be shown that if final quantitative results are required with a precision of $1 \%$ then mass absorption coefficients with a similar precision are necessary [1]. Unfortunately, the scatter in published mac data in literature is one to two orders of magnitude higher. It is out of the question to work with such a large scatter in vital physical input data in the matrix correction program. This is especially true, of course, for the absorption correction scheme. The latter has to be of outstanding reliability anyway in order to be able to cope with the extreme demands encountered in EPMA of ultra-light elements. We have pointed out [1] that the performance of a particular matrix correction procedure can only be judged in conjunction with consistent sets of mass absorption coefficients for each of the ultra-light elements. This assessment, in turn, can only be carried out when large databases of high-quality measurements are available over the widest possible range in experimental conditions.

Apart from the fundamental problems briefly mentioned so far there are also a number of practical problems during the actual analysis, some of which are not always easy to deal with. 
One of the items of major importance is the specimen preparation, in which topics such as flatness and cleanliness of the specimen surface play a crucial role. Due to the usually strong absorption the x-ray emission volume in the specimen will be extremely shallow. Hence, anything that interferes with this shallow volume rapidly leads to a deterioration of the results.

For the same reason contamination phenomena can have disastrous effects on the analysis. Not only the (well-known) effects of carbon contamination have to be considered but also those of the much less well-known contamination with oxygen [4]. Carbon contamination is not only very bad for the analysis of carbon but also for the analysis of nitrogen because $\mathrm{N}-\mathrm{K}_{\alpha} \mathrm{x}$-rays are heavily absorbed in carbon, due to the presence of the carbon K-edge. Especially during the relatively long time period required for an integral WD intensity measurement on the same location the build-up of carbon can have deleterious effects on the analysis. Several devices, such as a liquid nitrogen cooling trap or the even more effective air-jet, can be used to reduce the build-up of carbon considerably. One has to be cautious, though, that while using an air-jet the specimen is not oxidized under the electron beam. There are cases, notably with some nitrides [4], where even without air-jet a process of oxidation sets in immediately after the electron beam has been positioned on the specimen. These examples demonstrate again how important it is that the operator is fully aware of all the problems that might disturb the analysis of ultra-light elements and that he constantly monitors the signals of all the elements involved.

Another major problem is usually the correct determination of the background, which is all the more important in case of relatively low intensities. Typical problems are strong curvatures or kinks in the background or the presence of multitudes of higher-order metal lines, which interfere with the light element peak. These problems are most pronounced with a conventional lead-stearate crystal, which is very effective in transmitting higher-orders of reflections. In combination with its low peak intensities such a crystal usually produces very low peak-to-background ratios, especially in the case of nitrogen. Fortunately, the new synthetic multilayer crystals have proved to be a great help [2] in two ways: They can supply considerably higher peak count rates than the conventional stearate crystal (more than an order of magnitude is not uncommon) and they can suppress higher orders of reflection quite effectively. The latter effect has to be checked for each particular light element because it is dependent on the wavelength range and the $2 \mathrm{~d}$-spacing of the crystal. The poorer spectral resolution of these crystals appears to be only a small price to pay for all these benefits. In some cases though, e.g., in Nb-borides, their use can lead to a complete overlap of B-K $\mathrm{K}_{\alpha}$ and $\mathrm{Nb}-\mathrm{M}_{\zeta}$ peaks, which can normally be resolved fairly well with a lead stearate crystal.

\section{References}

[1] G.F. Bastin and H.J.M. Heijligers, in K.F.J. Heinrich and D.E. Newbury, Eds., Electron Probe Quantitation, Plenum, New York (1991), 145.

[2] G.F. Bastin and H.J.M. Heijligers, in D. Williams, J. Goldstein, and D. Newbury, Eds., X-Ray Spectrometry in Electron Beam Instruments, Plenum, New York (1995), 239.

[3] G. Wiech, "X-ray emission spectroscopy", NATO Adv. Study Inst., P. Day, Ed., Emission and Scattering Techniques Ser. C., (1981), 103.

[4] G.F. Bastin and H.J.M. Heijligers, Microbeam Analysis, D. E. Newbury, Ed., San Francisco Press, (1988), 325. 